\title{
Reduction and calibration of eye monitor data
}

\author{
REINHOLD KLIEGL and RICHARD K. OLSON \\ University of Colorado, Boulder, Colorado 80309
}

\begin{abstract}
The present paper presents FORTRAN programs for reducing eye monitor output to fixations and for mapping these fixations to locations in the stimulus space. Flexible parameters of the fixations program allow for determination of the beginning and end of fixations under different resolution criteria and for indicating loss of accurate measurement. The calibration program is based on a rectangular 9-point fixation grid. Each fixation is rescaled within this grid by solving for a quadratic equation. The rescaled values are output in a flexibly determined rectangular coordinate system that is related to the stimulus space, such as character position on the screen. The programs were developed for the 60-Hz Applied Sciences corneal reflection eye monitor, but they may be used with a number of other systems.
\end{abstract}

Technology for monitoring eye fixations has advanced rapidly over the past decade. Several commercial eye monitoring systems are now available that are capable of better than $1 \mathrm{deg}$ measurement accuracy and are relatively free of drift and other artifacts (Young \& Sheena, 1975). These systems operate at sampling rates of $60 \mathrm{~Hz}$ or higher, and their outputs are typically directed to a computer for storage or on-line stimulus control. The associated computer may also be used for calibration and the reduction of data to fixation location and duration. Calibration is necessary for many systems because their analog or digital output for eye position varies with individual differences in the shape of the eyes. Reduction of eye position data to fixation location and duration is often advantageous because it reflects some basic operating characteristics of the eye motor system, and it results in considerable savings in data storage and analysis.

Several eye fixation laboratories have developed reduction and calibration programs. Most of these are fundamentally similar. The programs used in our laboratory are written in RT-11 FORTRAN.

\section{REDUCTION PROGRAM}

The order of reducing and calibrating eye monitor data depends on the initial quality of the data and whether eye position is being used for on-line experimental control. If equal differences in degrees of eye position result in very unequal changes in analog or digital machine output in different regions of the visual field, then calibration should precede reduction to fixations. As explained below, new fixations are calculated

This research was supported by U.S. Public Health Service Grant HDMH1168101A1. Appreciation is expressed to Larry Bagget for working out the quadratic solution, to Steve Barlow for his geometric vision in Figure 2, and to Brian Davidson for his critical reading of the manuscript. Requests for reprints and program listings should be sent to Reinhold Kliegl or Richard Olson, Department of Psychology, University of Colorado, Boulder, Colorado 80309. when the index of eye position changes by a specified number of units. If the visual angle value of these units is quite different across the field, there will be a corresponding change in the window size for calculating a fixation, and the calibration should be performed directly on machine output prior to the reduction analysis. However, if the machine output is already reasonably well calibrated and it is not used for on-line control, then a reduction to fixations prior to the final calibration will require considerably less processing time. This is because the calibration is performed on the 3- to $5-\mathrm{Hz}$ fixations rather than the higher $\mathrm{Hz}$ machine output.

Our method for reducing data to fixations is similar to an algorithm first reported by Mason (1976). A fixation "window" is initially defined in the program, which determines the amount of change in machine units on the $\mathrm{X}$-axis (XDELTA) or Y-axis (YDELTA) that will indicate a change in eye position. Thus the window may take any rectangular form specified by the experimenter. By substituting one line in the program, any ellipsoid can be set up as a window with XDELTA and YDELTA as half-axes. The particular XDELTA and YDELTA values chosen depend on the noise characteristics of the machine and the nature of the task. Typically, the smallest values are chosen that exceed noise fluctuations in machine output during a fixation. In our reading research, we set XDELTA to 5 units and YDELTA to 9 units. The larger YDELTA value reflects a greater amount of machine noise on that axis. Also, fixations are ultimately allocated to line and letter position, with greater distance between lines on the $\mathrm{Y}$-axis than between letters on the $\mathrm{X}$-axis. Separate fixations within the window will be called a single fixation, and actual fixations may be broken into shorter segments if the window size is exceeded by machine noise.

The reduction program begins by calculating a running mean for $\mathrm{X}$ and $\mathrm{Y}$ eye position from successive samples. If a new sample deviated from the running 
Table 1

Transitions in Data From the Eye Monitor

\begin{tabular}{|c|c|c|c|}
\hline $\mathrm{X}$ & $\mathrm{Y}$ & Pupil & Transition Description \\
\hline 10 & 30 & 100 & \\
\hline 10 & 30 & 100 & \\
\hline 10 & 30 & 100 & \\
\hline 10 & 30 & 100 & \\
\hline 10 & 30 & 100 & A: Transition with no sample between fixations. \\
\hline 20 & 30 & 0 & \\
\hline 20 & 30 & 0 & \\
\hline 20 & 30 & 0 & \\
\hline 20 & 30 & 0 & \\
\hline 20 & 30 & 0 & \\
\hline 27 & 30 & 100 & B: Transition with one sample between fixations. \\
\hline 35 & 30 & 100 & \\
\hline 35 & 30 & 100 & \\
\hline 35 & 30 & 0 & \\
\hline 35 & 30 & 100 & \\
\hline 35 & 30 & 100 & \\
\hline 42 & 30 & 100 & C: Transition with two samples between fixations. \\
\hline 48 & 30 & 100 & \\
\hline 55 & 30 & 100 & \\
\hline 55 & 30 & 100 & \\
\hline 55 & 30 & 10 & \\
\hline 55 & 30 & 100 & \\
\hline 55 & 30 & 100 & \\
\hline 125 & 30 & 100 & D: One sample falls outside window; next sample is within window. \\
\hline 55 & 30 & 100 & \\
\hline 65 & 30 & 100 & \\
\hline 65 & 30 & 100 & \\
\hline 65 & 30 & 100 & \\
\hline 65 & 30 & 100 & \\
\hline 65 & 30 & 100 & \\
\hline 75 & 30 & 100 & \\
\hline 85 & 30 & 100 & E Second sample falls outside window at the beginning of a new fixation. \\
\hline 75 & 30 & 100 & \\
\hline 75 & 30 & 100 & \\
\hline 75 & 30 & 100 & \\
\hline
\end{tabular}

mean by more than the XDELTA or YDELTA values, it is stored. If the second sample falls within the XDELTA and YDELTA limits, the deviant value is assumed to reflect machine noise rather than a change in eye position. This is a reasonable assumption from the known parameters of eye movements and fixations, since fixations shorter than $50 \mathrm{msec}$ are rarely observed. Thus a single deviant sample representing $17 \mathrm{msec}$ in a $60-\mathrm{Hz}$ system could not indicate a separate fixation.

With some eye monitor systems it is advantageous to allow more than one deviant sample before calculating a new fixation. With the Applied Sciences monitor, a noise filter allowing one deviant sample seems to be sufficient and we rarely observe two successive noise events outside the window. The Applied Sciences monitors may do some noise filtering by averaging from two to four television frames for each output. This reduces noise in the signal, but it also distorts the true eye position during moves and at the beginning and end of fixations. Therefore, we disabled this averaging function and filter the data in the reduction program.

The reduction program functions may be clarified by reference to Table 1 and Table 2. Raw data for X-axis position in machine units are simulated in the left column of Table 1. Y-axis position and pupil diameter may be ignored for now. Six columns at the bottom of Table 2 indicate the output of the reduction program for these raw data. Later, we will discuss the separation of samples in reduced output representing eye movements and true fixations. Letters A-E mark different types of transitions in the raw data and their representation in the reduced data, with XDELTA set at 5 . At $60 \mathrm{~Hz}$, short eye movements sometimes result in two longer adjacent fixations with no samples indicating a move

Table 2 Reduced Data

\begin{tabular}{cccccc}
\hline $\begin{array}{c}\text { Number } \\
\text { of Sam- } \\
\text { ples }\end{array}$ & $\begin{array}{c}\text { Reduc- } \\
\text { tion } \\
\text { Codes }\end{array}$ & $\begin{array}{c}\text { X } \\
\text { Posi- } \\
\text { tion }\end{array}$ & $\begin{array}{c}\text { Y } \\
\text { Posi- } \\
\text { tion }\end{array}$ & $\begin{array}{c}\text { Average } \\
\text { Pupil }\end{array}$ & $\begin{array}{c}\text { Transi- } \\
\text { tion Con- } \\
\text { dition }\end{array}$ \\
\hline 5 & 3 & 10 & 30 & 100 & A \\
5 & 54 & 20 & 30 & 0 & \\
1 & 2 & 27 & 30 & 100 & B \\
5 & 54 & 35 & 30 & 80 & \\
1 & 2 & 42 & 30 & 100 & C \\
1 & 0 & 48 & 30 & 100 & D \\
7 & 63 & 55 & 30 & 85 & \\
5 & 4 & 65 & 30 & 100 & \\
1 & 2 & 75 & 30 & 100 & \\
1 & 0 & 85 & 30 & 100 & E \\
3 & 0 & 75 & 30 & 100 & \\
\hline
\end{tabular}


between them, as in A. B indicates a single sample transition between two fixations. The $\mathrm{C}$ transition consists of two samples. These are marked in the reduced data as two separate fixations, since their separation exceeds an XDELTA of 5 . If the difference was 5 units or less, they would have been combined as a single fixation with a duration of two samples and an average position of $45 . D$ indicates the case of a single sample falling outside the window when the next sample is within the window. In this situation, the position value of the outlier is ignored in the fixation position calculation, but it is included in the estimate of fixation duration.

$E$ indicates a special case in which a noise event would be wrongfully excluded from a fixation. The first sample of 75 might represent the beginning of a fixation, but the subsequent noise event precludes the inclusion of either signal in the fixation. This leads to an underestimation of fixation duration. If such noise events are frequent at the beginning of fixations, a secondary reduction program should look for these events and include them in the appropriate fixation. In addition, the secondary reduction should combine parts of fixations that have been broken up by noise events. Programs for this purpose are described by Kliegl (1981).

The Applied Sciences eye monitor also measures pupil diameter. This output is simulated in the third column of raw data. Pupil diameter may be related to the psychologically interesting variables of processing load and arousal level, but it may also be used to indicate blinks and loss of accurate measurement. It is the latter property that is very useful in the context of this program. A flexible parameter (PDELTA) is set to define the largest percent reduction in diameter between successive fixations that will not result in that fixation's being flagged. In the present simulation, PDELTA is 15. Three different types of pupil events are embedded in the raw data. The first sequence of zeros occurs during the second fixation. In the reduction program output, the average pupil diameter is zero, and the value 50 is added to the number in the reduction code column. (The second integer in the column is a marker for internal program calculations that will not be discussed here.) The second pupil diameter change is a zero for a single sample among the five samples of a fixation. Thus the average diameter for this fixation is 80 , which is below the $15 \%$ reduction limit, and this fixation is also flagged by adding 50 to the reduction code column. In the third case, pupil diameter goes to 10 for one sample in a fixation with seven samples. When one or more samples fall below the reduction limit, but the average fixation pupil diameter is above the limit, 60 is added to the reduction code column.

The simulated raw data do not accurately indicate what would happen with the described fluctuations in pupil diameter. Changes of this size are considerably beyond the range of actual pupil constriction, and the output reflects a loss of pupil position caused by a blink or by machine problems. As a result, the $\mathrm{X}$ and $\mathrm{Y}$ posi- tion values are very different from true eye position. Thus these data must be flagged by pupil diameter changes for marking as blinks in the secondary reduction program described by Kliegl (1981).

The process of calibrating and mapping the reduced machine unit data to stimulus positions such as lines and letter spaces is described in the following section.

\section{CALIBRATION PROGRAM}

The purpose of the calibration program is to relate the output of the eye monitor to eye fixations at locations in the stimulus space. This is achieved by first having the subject fixate nine points in a rectangular calibration grid. In our reading experiments, the center point is located at the middle of the stimulus screen and the four corners correspond to boundaries of the stimulus field. Two assumptions are important: The subject must accurately fixate the calibration points, and the eye monitor output should be linearly related to visual direction between the calibration points. The assumption of accurate calibration fixations is probably valid for cooperative subjects fixating a small point, under the precautions suggested by McConkie (1981). The assump. tion of linear interpolation between calibration points may also be met within reasonable limits for most subjects and viewing conditions. Video-based systems that track the relative positions of the pupil and a corneal reflection may sometimes produce curvilinear output between points, but as our checks for calibration accuracy will show, these deviations from linearity are usually not very great.

Although the output of eye tracking devices typically has a systematic relationship to eye position, the geometric nature of this relation can be rather complex. Figure 1 presents a spatial mapping of hypothetical

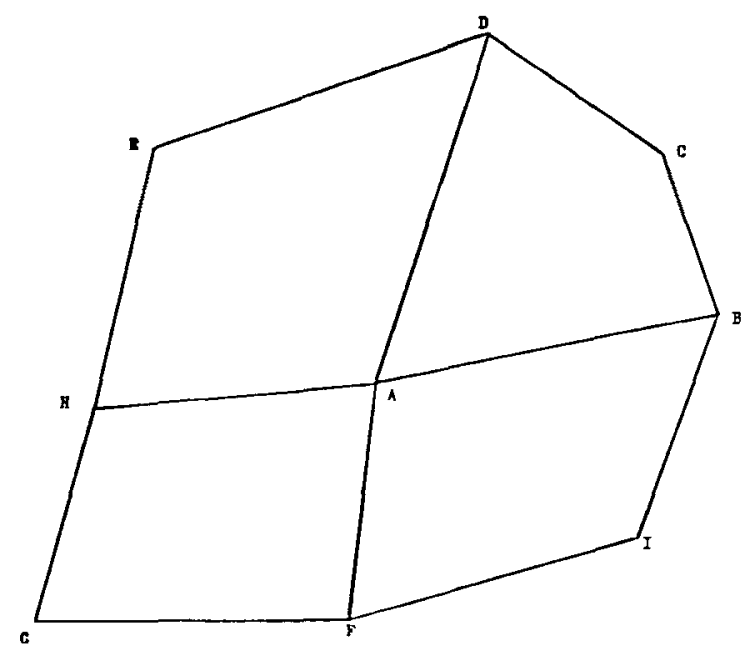

Figure 1. The figure represents the spatial mapping of hypothetical machine output for a subject's fixations on a 9-point calibration grid. 
machine output for a subject's fixations on a 9-point rectangular calibration grid. Of course, the degree of distortion will vary with different systems. The distortion in this example is considerably greater than the precalibrated output of our Applied Sciences Model 1996. Therefore, we are able to reduce the data to fixations before our calibration.

The first step in using the fixation grid output to calibrate subsequent fixations on stimuli is to determine the appropriate quadrant for the current data point. The program does this by calculating the geometric position of the extended lines $\mathrm{AB}, \mathrm{AH}, \mathrm{AF}$, and $\mathrm{AD}$ in Figure 1. Then a stepping algorithm determines whether the data point is above or below each of these lines, thereby uniquely defining the extended quadrant to be used for calibrating that point.

Figure 2 represents a data point $P$ in Quadrant $A B C D$. The calibration of this point into a rectangular space is accomplished by solving the quadratic equations in the Appendix. In actual practice, all of the terms except for $\left(m_{1}-a_{1}\right)$ and $\left(m_{2}-a_{2}\right)$ are initially computed from the fixation grid output and are entered as constants in a table. The subsequent use of these tabled values saves considerable computation time for each new data point.

It is easy to see the functional operation of the quadratic solutions by referring to Figure 2 . First, a linear shift is performed to place the $A B C D$ quadralateral with $A=A^{\prime}$ at the origin of the rectangular axes $X^{\prime}$ and $Y^{\prime}$. Second, Focal Point $F 1$ is established from the slopes of Lines $A D$ and $B C$, and Focal Point $F 2$ is established from the slopes of $A B$ and DC. Intercepts of Lines $C D$ and $C B$ yield $D^{\prime}$ on the $Y^{\prime}$-axis and $B^{\prime}$ on the $X^{\prime}$-axis. Now a rectangle $A^{\prime} B^{\prime} C^{\prime} D^{\prime}$ has been defined. In the third step, intercepts of lines through $P$ from $F 1$ and $F 2$ are calculated for the $X$ - and $Y$-axes, yielding $P^{\prime}{ }_{X}$ and

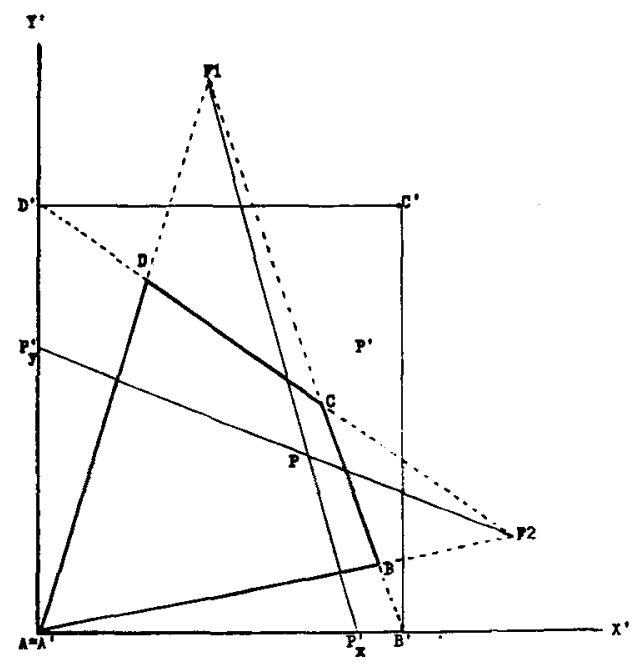

Figure 2. A geometric representation of the quadratic solution for mapping Point $P$ in the machine output for Quadrant $A B C D$ into $P^{\prime}$ in rectangular quadrant $A^{\prime} B^{\prime} C^{\prime} D^{\prime}$.
Table 3

Absolute Mean (M) and Range (R) of Deviations From Assumed Fixation Location

\begin{tabular}{|c|c|c|c|c|c|c|c|c|c|c|}
\hline \multirow{3}{*}{$\begin{array}{l}\text { Line } \\
\text { Position }\end{array}$} & \multicolumn{10}{|c|}{ Character Position } \\
\hline & \multicolumn{2}{|c|}{10} & \multicolumn{2}{|c|}{25} & \multicolumn{2}{|c|}{40} & \multicolumn{2}{|c|}{55} & \multicolumn{2}{|c|}{60} \\
\hline & $\mathbf{M}$ & $\mathbf{R}$ & $\mathbf{M}$ & $\mathbf{R}$ & $\mathbf{M}$ & $\mathbf{R}$ & $\mathbf{M}$ & $\mathbf{R}$ & $\mathbf{M}$ & $\mathbf{R}$ \\
\hline 1 & .0 & 0 & .2 & 1 & .3 & 1 & .1 & 1 & 1.0 & 2 \\
\hline 2 & .4 & 1 & .1 & 1 & .5 & 1 & .2 & 1 & .5 & 1 \\
\hline 3 & .2 & 1 & .0 & 0 & .2 & 1 & .2 & 1 & .3 & 1 \\
\hline 4 & .4 & 1 & .2 & 1 & .3 & 1 & .3 & 1 & .3 & 1 \\
\hline 5 & .7 & 1 & .4 & 2 & .8 & 2 & .1 & 1 & .0 & 0 \\
\hline 6 & .3 & $l$ & .4 & 2 & .7 & 2 & .1 & 1 & .6 & 1 \\
\hline 7 & .5 & 1 & .1 & 1 & .3 & 1 & .2 & 1 & .3 & 1 \\
\hline 8 & .8 & 2 & .4 & 1 & .2 & 2 & .4 & $i$ & .1 & 1 \\
\hline 9 & .6 & 1 & .2 & 1 & .2 & 1 & .4 & 1 & .5 & 1 \\
\hline 10 & .7 & 1 & .5 & 1 & .6 & 1 & .3 & 1 & .5 & 1 \\
\hline 11 & .2 & 1 & .2 & 1 & .4 & 1 & .4 & 1 & .3 & 1 \\
\hline
\end{tabular}

Note-Deviation and range are in character position units, which subtend about $.33 \mathrm{deg}$ of visual angle. Italicized values are calibration points.

$\mathbf{P}^{\prime}{ }_{\mathrm{y}}$, which define the calibrated location $\mathbf{P}^{\prime}$ in the rectangular space. However, the rectangle $A^{\prime} B^{\prime} C^{\prime} D^{\prime}$ and $P^{\prime}$ are still in arbitrary machine-related units. These values are rescaled to user-defined values XSCALE and YSCALE by a linear transformation. We use 61 character spaces horizontally and 11 lines vertically. Thus for one quadrant, XSCALE is set to 30 and YSCALE is set to 5.

McConkie (1981) has noted the need for adequate reporting of data quality from eye monitors. In Table 3 , the results for a test of calibration accuracy and repeatability are presented. The test was performed by first calibrating a subject on the 9-point grid. Then the subject completed 10 trials of fixations on a 55-point test grid over a period of $15 \mathrm{~min}$. Nine of these test fixation points, italicized in Table 3, were in the same location as the original calibration fixation points. Therefore, the data for these points represent estimates of accuracy and stability that are largely independent of calibration interpolation. The other points are all between the calibration fixation points and are therefore based on the assumption of linear interpolation in the calibration program. The correctness of this assumption is tested by comparing accuracy for the calibration fixation points with the 46 points in between.

The numbers in Table 3 represent the absolute mean and range of variability for computed fixation location from the assumed fixation on actual stimulus location. Each unit represents one character position, or about $.33 \mathrm{deg}$ of visual angle on the horizontal axis. There was no error for separation of lines of fixation points, so the vertical position data are not reported here. The mean absolute error for the nine calibration points was .42 , compared with .34 for the 46 interpolated points. As the range values suggest, $64 \%$ of the fixations were assigned to the appropriate character space, $36 \%$ were one character off, and .14\% were two characters off. Thus we can be reasonably sure that this subject's fixation data are accurate within one character space of the 
assumed target, under the experimental conditions of this test.

\section{CONCLUSION}

Two programs have been described that reduce eye monitor data to fixation location and duration and calibrate the fixation data. The reduction program provides sample clusters with pupil diameter condition codes that can be used in subsequent programs to separate fixations from eye movements and define blinks or bad data. The calibration program solves a quadratic equation to map fixations into the stimulus space.

Our experience and the described test have demonstrated the value of these programs for reducing and calibrating eye monitor data. These programs have been used to evaluate eye fixations in reading, but they can also be used for analyzing eye fixations on other stimuli.

\section{Program Requirements}

These programs run on a PDP-11/03 microcomputer with $28 \mathrm{~K}$ memory under the RT-11 operating system. They are written in RT-11 FORTRAN and can easily be adapted for use on other systems.

\section{Availability}

A printed listing of the programs may be obtained by writing to one of the authors.

\section{REFERENCES}

KLIEGL, R. Automated and interactive analysis of eye fixation data in reading. Behavior Research Methods \& Instrumentation, 1981, 13, 115-120.

MAson, R. L. Digital computer estimation of eye fixations. Behavior Research Methods \& Instrumentation, 1976, 8, 185-188.

McConkı, G. W. Evaluating and reporting data quality in eye movement research. Behavior Research Methods \& Instrumentation, 1981, 13, 97-106.

Young, L. R., \& Sheena, D. Survey of eye movement recording techniques. Behavior Research Methods \& Instrumentation, $1975,7,397-429$.

Appendix

Equations Used to Match Points of a Quadralateral Onto Corresponding Points in a Rectangle

Let $A B C D$ be a quadralateral with coordinates $A\left(a_{1}, a_{2}\right), B\left(b_{1}, b_{2}\right)$, etc.

Let $M\left(m_{1}, m_{2}\right)$ be a point in this quadralateral.

Let $A^{\prime} B^{\prime} C^{\prime} D^{\prime}$ be a rectangle with $A^{\prime}$ in the origin, $B^{\prime}$ at $(g, 0), C^{\prime}$ at $(g, h)$, and $D^{\prime}$ at $(0, h)$.

Let $S\left(s_{1}, s_{2}\right)$ be the corresponding point to $M$.

The following equations allow the calculation of $s_{1}$ and $s_{2}$, given $\mathrm{ABCD}, \mathrm{g}, \mathrm{h}$, and $\mathrm{M}$ :

$$
\begin{aligned}
& p_{1} s_{1}^{2}+q_{1} s_{1}+r_{1}=0 \\
& p_{2} s_{2}^{2}+q_{2} s_{2}+r_{2}=0
\end{aligned}
$$

where:

$$
\begin{aligned}
& u=1 /(g h) \\
& p_{1}=(u / g)\left[\left(b_{2}-a_{2}\right)\left(a_{1}-b_{1}+c_{1}-d_{1}\right)-\left(b_{1}-a_{1}\right)\left(a_{2}-b_{2}+c_{2}-d_{2}\right)\right] \\
& q_{1}=u\left\{\left[\left(m_{1}-a_{1}\right)\left(a_{2}-b_{2}+c_{2}-d_{2}\right)-\left(m_{2}-a_{2}\right)\left(a_{1}-b_{1}+c_{1}-d_{1}\right)\right]-\left[\left(b_{1}-a_{1}\right)\left(d_{2}-a_{2}\right)-\left(b_{2}-a_{2}\right)\left(d_{1}-a_{1}\right)\right]\right\} \\
& r_{1}=(1 / h)\left[\left(m_{1}-a_{1}\right)\left(d_{2}-a_{2}\right)-\left(m_{2}-a_{2}\right)\left(d_{1}-a_{1}\right)\right] \\
& p_{2}=(u / h)\left[\left(d_{2}-a_{2}\right)\left(a_{1}-b_{1}+c_{1}-d_{1}\right)-\left(d_{1}-a_{1}\right)\left(a_{2}-b_{2}+c_{2}-d_{2}\right)\right] \\
& q_{2}=u\left\{\left[\left(m_{1}-a_{1}\right)\left(a_{2}-b_{2}+c_{2}-d_{2}\right)-\left(m_{2}-a_{2}\right)\left(a_{1}-b_{1}+c_{1}-d_{1}\right)\right]+\left[\left(b_{1}-a_{1}\right)\left(d_{2}-a_{2}\right)-\left(b_{2}-a_{2}\right)\left(d_{1}-a_{1}\right)\right]\right\} \\
& r_{2}=(1 / g)\left[\left(m_{1}-a_{1}\right)\left(b_{2}-a_{2}\right)-\left(m_{2}-a_{2}\right)\left(b_{1}-a_{1}\right)\right]
\end{aligned}
$$

Note-If $\left(a_{1}-b_{1}+b_{1}-d_{1}\right)=0$ and $\left(a_{2}-b_{2}+c_{2}-d_{2}\right)=0$, then ABCD is a parallelogram. In this case, $p_{1}, p_{2}$, the first part of $q_{1}$, and the first part of $q_{2}$ are 0 and Equations 1 and 2 lose their quadratic terms. 